\title{
Assessment of Left Ventricular Functions in Hypertensive Diabetic Patients by Speckle Tracking Imaging: Correlation with Brain Natriuretic Peptide Levels
}

\author{
Mohammed Fahmy El-Noamany ${ }^{1}$, Ashraf Abd EIRaouf Dawood ${ }^{2}$, Waleed Abdou Ibrahim Hamed ${ }^{1}$, \\ Nowrus Emad Amer ${ }^{3, *}$ \\ ${ }^{1}$ Cardiology Department, Menoufia University, Menoufia, Egypt \\ ${ }^{2}$ Biochemistry Department, Menoufia University, Menoufia, Egypt \\ ${ }^{3}$ Cardiology Department of Students' Hospital, Menoufia University, Menoufia, Egypt
}

Email address:

mnoamany@hotmail.com (M. F. El-Noamany), kingashrafdawood@yahoo.com (A. A. E. Dawood), waleedabdou@yahoo.com (W. A. I. Hamed), nowrus.emad@gmail.com (N. E. Amer)

${ }^{*}$ Corresponding author

\section{To cite this article:}

Mohammed Fahmy El-Noamany, Ashraf Abd ElRaouf Dawood, Waleed Abdou Ibrahim Hamed, Nowrus Emad Amer. Assessment of Left Ventricular Functions in Hypertensive Diabetic Patients by Speckle Tracking Imaging: Correlation with Brain Natriuretic Peptide Levels. Cardiology and Cardiovascular Research. Vol. 4, No. 3, 2020, pp. 131-145. doi: 10.11648/j.ccr.20200403.19

Received: July 24, 2020; Accepted: August 10, 2020; Published: August 18, 2020

\begin{abstract}
Background: Hypertension and diabetes are major contributors to structural changes including myocardial fibrosis and progressive alteration of LV systolic and diastolic functions. Two-dimensional speckle tracking echocardiography is a reliable imaging modality that overcomes the limitations of Tissue Doppler imaging and allows a more accurate assessment of myocardial deformation at the global and regional levels. Objectives: The investigation aims to assess Left Ventricular functions in patients with hypertension and/or diabetes who have apparently preserved LV systolic function; using speckle tracking echocardiography, and correlate the findings with plasma Brain Natriuretic Peptide levels. Methods: Twenty healthy subjects were enrolled as a control group. Sixty patients with hypertension and/or diabetes were recruited and assembled as three equal groups, hypertensives, diabetics, and hypertensive-diabetics. 2D-STE was performed to assess LV longitudinal strain and strain rate. Plasma BNP levels were measured for all subjects. Results: Global systolic longitudinal strain was significantly reduced in patients compared to controls $(\mathrm{P}=0.001)$. It was more reduced in group 4. Global systolic $\mathrm{SR}$ was reduced in patients compared to controls, being more significantly reduced in groups 2 and $4(\mathrm{P}=0.001)$. Global early diastolic $\mathrm{SR}$ was reduced in patients compared to controls $(\mathrm{P}=0.001)$. Meanwhile, Global late diastolic $\mathrm{SR}$ values were higher among patients, especially groups 2 and $4(\mathrm{P}=0.001)$. $\mathrm{BNP}$ levels were significantly higher in group 4 compared to group $1(\mathrm{P}=0.000)$, group $2(\mathrm{P}=0.000)$, and group $3(\mathrm{P}=0.000)$. $\mathrm{BNP}$ levels and global systolic strain in group 4 were significantly correlated. Conclusion: LV systolic dysfunction was found among all patients groups, confirmed by the significant reduction in LV global longitudinal systolic SR. Hypertensive-diabetics exhibited lower global strain than patients with hypertension only and patients with diabetes only, even though their EF showed no apparent difference. Elevated BNP levels, being the highest among group 4 , indicate the presence of ventricular dysfunction, even before chamber failure occurs.
\end{abstract}

Keywords: Hypertension, Diabetes, Speckle Tracking Echocardiography, Left Ventricular Functions, Strain, Strain Rate

\section{Introduction}

Hypertension is a heterogeneous disorder with a number of well defined, putative etiologies. The World Health Organization estimates that hypertension may cause 7.1 million premature deaths and $4.5 \%$ of the disease burden worldwide [1]. Hypertension is a major risk factor for stroke and major cardiovascular diseases (CVD) and is thus 
associated with significant cardiovascular morbidity and mortality. It is also the most common cause of heart failure with reduced LVEF.

Diabetes Mellitus (DM) is the most common metabolic disease, especially in Western countries, and its prevalence will double in the next 25 years [2]. Cardiovascular diseases are the leading cause of death among diabetic patients because, as widely demonstrated, chronic hyperglycemia results in morphological and functional changes on vascular walls, which is likely to lead to the development of atherosclerotic plaque [3].

Diabetes and hypertension frequently occur together, there is substantial overlap between both diseases, reflecting considerable overlap in their etiology and disease mechanisms. In the US population, hypertension occurs in approximately $30 \%$ of patients with type 1 diabetes, and in $50 \%$ to $80 \%$ of patients with type 2 diabetes [4]. In reality, diabetes and hypertension are found in the same individual more often than would occur by chance, whereas the overlap between dysglycemia and raised blood pressure is even more substantial than that between diabetes and hypertension [5].

The fact that hypertension and diabetes often share comorbidities and conditions -like obesity and LV hypertrophy- that can impact LV structure and mechanics, makes it difficult to quantify the individual and synergistic roles of diabetes and hypertension on LV diastolic function [6].

The finding of a higher LV end-diastolic pressure when diabetes and hypertension coexist, compared with either condition alone, could explain in part the additional risk of developing heart failure in patients with combined diabetes and hypertension compared with patients with hypertension alone [7-9].

BNP is produced by cardiac ventricular cells in response to volume expansion and increased pressure load [10]. It acts mainly via natriuretic peptide receptors (NPRs) that are present in large vessels and kidneys. Once stimulated, NPRs promote diuresis, natriuresis, and vasodilation and inhibit the renin-angiotensin-aldosterone system.

The role of BNP as a prognostic risk marker for CVD has been investigated in patients with chronic heart failure and acute myocardial infarction, showing an increased risk of future CVD morbidity or mortality with elevated plasma levels of BNP.

Measurement of plasma NT-proBNP seems to provide the same information as that of plasma BNP [11]. Upregulation of BNP expression is widely used as a diagnostic marker for LV hypertrophy, diastolic dysfunction, and heart failure in the clinic. Studies have postulated that BNP serves as an endogenous brake on the LV myocardium, seeking to curb the runaway train of signaling pathways that drive the progression from LV hypertrophy through remodeling, heart failure, and death [12].

Both, BNP and the amino-terminal fragment of the precursor peptide (NT-proBNP) are used as prognostic and diagnostic markers in cardiovascular abnormalities such as hypertension, left ventricular diastolic dysfunction, and HF [13]. It has been reported that plasma BNP concentration is correlated with LV mass index in patients with hypertension, where LV hypertrophy is a potent risk factor for cardiovascular morbidity and mortality. Since impaired relaxation usually precedes reduced EF, elevated BNP concentrations can detect impaired relaxation with good sensitivity and specificity [14]. Plasma levels of BNP and NT-proBNP also correlate with left ventricular dilatation, remodeling, and dysfunction [15]. In addition to that, plasma NT-proBNP has been shown to be a strong risk marker for cardiovascular disease and congestive heart failure in patients with diabetes and generalized vascular damage as estimated from the presence of microalbuminuria [16].

Speckle-tracking echocardiography (STE) is a noninvasive, angle-independent technique where myocardial features are kept temporally stable throughout the cardiac cycle and unique for each myocardial region independent from cardiac translational movements [17]. Analysis of both longitudinal strain and SR increases the sensitivity of early detection of subclinical cardiac involvement in various conditions such as amyloidosis and systolic dysfunction in patients with preserved LVEF [18].

Conventional echocardiography detects abnormalities in LV systolic function only in the advanced stages of hypertensive heart disease (HHD), when a clear LV remodeling/hypertrophy is evident, also, isolated abnormalities of diastolic performance are rare, and they're most frequently associated with a subclinical impairment of systolic function [19].

STE provides more information than Tissue Doppler imaging (TDI), allowing a non-invasive measurement of overall LV strain and twist. It can provide mechanistic insight into systolic dysfunction even in patients without structural cardiac alterations [20].

In hypertensive patients with normal LV geometry, impairment of longitudinal systolic function has been detected, besides the preclinical systolic dysfunction that occurs in hypertensive patients with LV hypertrophy [21]. Thus, STE is more sensitive than both conventional echocardiography and TDI in identifying the reduction of intrinsic myocardial contractility, evident in hypertensive patients, long before LV hypertrophy becomes detectable. 2D-STE also provides useful information about the development of subclinical myocardial dysfunction in a diabetic setting before the overt appearance of diabetic cardiomyopathy [22].

This study was designed to assess Left Ventricular functions in patients with hypertension and diabetes; using speckle tracking echocardiography, and correlate the findings with Brain Natriuretic Peptide levels.

\section{Methods}

\subsection{Study Population \& Grouping}

The study was carried out on 60 patients who were Hypertensive or Diabetic or both, as well as 20 healthy subjects who were recruited from the hospitals' cardiology 
outpatient clinics. The study protocol was approved by the local Ethical Committee and was in agreement with the "World Medical Association Declaration of Helsinki". An informed verbal and written consent for accepting the participation in the study was obtained from every participating patient.

Control volunteers were normotensive non-diabetic individuals, who were free from any cardiovascular risk factors. Hypertensive patients were diagnosed based on ESH/ESC guidelines for the management of hypertension if SBP $\geq 140 \mathrm{mmHg}$ and/or DBP $\geq 90 \mathrm{mmHg}$ on two or more hospital visits at one-week interval. Diabetic patients were diagnosed based on the criteria set by the American Diabetes Association; Fasting Plasma Glucose $\geq 126 \mathrm{mg} / \mathrm{dl} \quad(7.0$ $\mathrm{mmol} / \mathrm{l})$ or Two-hour Plasma Glucose $\geq 200 \mathrm{mg} / \mathrm{dl} \quad(11.1$ $\mathrm{mmol} / \mathrm{l}$ ) during an Oral Glucose Tolerance Test.

Groups Included:

Group 1: Included 20 age and sex-matched healthy individuals.

Group 2: Included 20 hypertensive, non-diabetic patients.

Group 3: Included 20 diabetic, non-hypertensive patients.

Group 4: Included 20 hypertensive-diabetic patients

\subsection{Exclusion Criteria}

Patients with impaired left ventricular systolic function (ejection fraction $<50 \%$ ) or with signs or clinical symptoms of heart failure, patients who had known coronary artery disease, patients with significant valvular disease, patients with atrial fibrillation, premature ventricular contractions (PVCs), heart block or other rhythm disturbances, patients with pericardial diseases or congenital heart diseases, patients with secondary causes of hypertension, and patients with unsatisfying echocardiographic images.

\subsection{Methods}

All patients had their complete clinical history taken. They were also subjected to thorough general and cardiac examination.

\subsubsection{Clinical \& Laboratory Evaluation}

A thorough history was obtained, including personal \& family history, history of hypertension, diabetes, and other diseases. A proper general examination was done assessing the general condition, arterial pulse, measurement of systemic blood pressure. Anthropometric measures were obtained, including measurement of body weight in kilograms and height in meters from which the body mass index $(\mathrm{BMI})$ was calculated $\left(\mathrm{BMI}=\right.$ Weight $(\mathrm{kg}) / \mathrm{Height}^{2}$ $\left.\left(\mathrm{m}^{2}\right)\right)$.

A complete cardiac examination was performed in addition to resting standard 12 leads electrocardiogram.

Total cholesterol levels were measured in all patients as well as the level of plasma BNP which was analyzed using BNP-32 (Human) Kit (ELISA).

\subsubsection{Conventional Echocardiography}

Conventional echocardiographic Doppler study, as well as
Tissue Doppler imaging and 2D speckle tracking, were performed using Vivid 9, General Electric Healthcare (GE Vingmed, Norway) equipped with a harmonic M5S variablefrequency (1.7-4 MHz) phased-array transducer.

All subjects were examined in the left lateral decubitus position according to the recommendations of the American Society of Echocardiography and connected to single-lead ECG [23].

LV dimensions were obtained using M-mode echocardiography. Measurements were obtained from the left parasternal view at the end-diastole using the widest LV cavity diameter, and at the end-systole using the narrowest LV cavity diameter. These measurements included: Enddiastolic thickness of the ventricular septum (IVSd), enddiastolic thickness of the LV posterior wall (PWd), enddiastolic LV diameter (LVEDd), end-systolic LV diameter (LVEDs), Left Atrial dimensions (LA), Aortic root dimensions (AO), LV fractional shortening (FS\%) and $\mathrm{LV}$ ejection fraction (EF\%). Pulsed wave Doppler (PW) echocardiography was used to evaluate LV diastolic function; recordings were obtained from apical 4 chamber view, peak waves of both early diastolic filling (E), and late diastolic filling (A) were measured, and E/A ratio was calculated.

Tissue Doppler imaging was performed by activating the pulsed wave tissue Doppler function in the apical 4-chamber view while positioning the sample volume $5 \mathrm{~mm}$ over the septal and lateral mitral annuli. Peak systolic $\left(\mathrm{S}^{\prime}\right)$, early diastolic $\left(\mathrm{e}^{\prime}\right)$, and late diastolic $\left(\mathrm{a}^{\prime}\right)$ velocities over three consecutive cardiac cycles were measured. The average $\mathrm{e}^{\prime}$ velocity at septal and lateral mitral annuli was estimated and the $\mathrm{E} / \mathrm{e}^{\prime}$ ratio was calculated.

\subsubsection{D-Speckle Tracking Echocardiography}

Image analysis was performed offline on a personal computer (PC) workstation using custom analysis software (Echopac PC, Version 1.8.1. X, GE Healthcare). Longitudinal strain and strain rate were assessed in the $6 \mathrm{LV}$ walls and the software algorithm automatically segmented the LV into 18 equidistant segments model in a 'bull's eye' plot, and each segment was individually analyzed. The average value of strain (\%) at each segment (basal, mid, and apical) and global LV strain obtained from averaging the strain values of $18 \mathrm{LV}$ segments were calculated and used for comparisons between control and the hypertensive, diabetic, hypertensive diabetic groups.

The average value of peak systolic SR $\left(\mathrm{SRs} \mathrm{s}^{-1}\right)$, peak early diastolic SR (SRe s $\left.{ }^{-1}\right)$, peak late diastolic SR (SRa s $\left.{ }^{-1}\right)$ at each segment (basal, mid, and apical), as well as global LV systolic and diastolic SR obtained from averaging the peak values of $18 \mathrm{LV}$ segments, were calculated and used for comparisons between the control group and patients groups.

\subsubsection{Plasma BNP Levels Measurement}

All samples were collected by venipuncture into redcapped tubes.

Plasma was separated by centrifugation, stored at $-70 \mathrm{c}$. Analysis was done using the BNP-32 (Human) Kit (ELISA). 


\section{Statistical Analysis}

The collected data has been tabulated and statistically analyzed by SPSS statistical package on IBM compatible computer [24].

Statistical presentation and analysis of the study have been conducted using descriptive statistics: e.g. mean $(\bar{X})$ and standard deviation (SD), as well as Analytic statistics: e.g. Ftest (ANOVA-analysis of variance) and Tukey's honest significant difference test.

Qualitative data has been expressed as number and percentage (No \& \%) and analyzed by applying the Chisquare test $\left(\mathrm{X}^{2}\right)$.

The Pearson Linear correlation coefficient ( $r$ ) was used to measure of the strength of a linear association between two variables, and Receiver Operating Characteristic (ROC) was used to evaluate the performance of diagnostic tests and more generally for evaluating the accuracy of a statistical model that classifies subjects into 1 of 2 categories, diseased or nondiseased, it was used to determine the optimal cutoff point of peak systolic strain, systolic SR, early and late diastolic SR as measurements for LV dysfunction that would differentiate between patients and controls.

\section{Results}

\subsection{Demographic, Clinical and Laboratory Data}

Table 1 shows that there was a highly statistically significant difference between the control group and all patients groups regarding their Body mass index. The mean values of total cholesterol level were relatively lower in the control group, compared to the HTN group, DM group, and HTN-DM group where the mean values of total cholesterol level were somewhat close in all 3 groups.

Table 1. Comparison between the study groups regarding demographic, clinical, and laboratory data.

\begin{tabular}{llllll}
\hline \multirow{2}{*}{ Items } & & Group & & \\
\cline { 3 - 6 } & & Control (n=20) & HTN (n=20) & DM (n=20) & HTN - DM (n=20) \\
\hline Age $($ Years) & Mean \pm SD & $32.25 \pm 12.58$ & $54.15 \pm 10.06$ & $52.05 \pm 7.79$ & $50.05 \pm 10.18$ \\
Weight $(\mathrm{Kg})$ & Mean \pm SD & $71.30 \pm 14.03$ & $79.60 \pm 10.83$ & $84.50 \pm 13.95$ & $79.85 \pm 15.45$ \\
BMI & Mean \pm SD & $22.12 \pm 1.36$ & $23.4 \pm 1.69$ & $25.0 \pm 2.08$ & $24.42 \pm 1.96$ \\
SBP $(\mathrm{mmHg})$ & Mean \pm SD & $114.50 \pm 6.06$ & $144.00 \pm 16.67$ & $126.50 \pm 9.33$ & $138.00 \pm 13.22$ \\
DBP (mmHg) & Mean \pm SD & $70.00 \pm 7.25$ & $89.50 \pm 9.45$ & $82.00 \pm 7.68$ & $86.50 \pm 7.45$ \\
Pulse $(\mathrm{bpm})$ & Mean \pm SD & $77.6 \pm 8.76$ & $77.05 \pm 8.36$ & $80.00 \pm 9.61$ & $78.40 \pm 8.79$ \\
Total Cholesterol $(\mathrm{mg} / \mathrm{dl})$ & Mean \pm SD & $167.45 \pm 11.46$ & $182.70 \pm 17.00$ & $181.9 \pm 18.54$ & $181.40 \pm 17.53$ \\
BNP $(\mathrm{pg} / \mathrm{ml})$ & Mean \pm SD & $35.50 \pm 6.46$ & $74.55 \pm 8.90$ & $99.25 \pm 12.47$ & $129.25 \pm 13.46$ \\
\hline
\end{tabular}

Table 1. Continued

\begin{tabular}{|c|c|c|c|c|c|}
\hline \multirow{3}{*}{ Items } & \multirow{2}{*}{ ANOVA } & & \multicolumn{3}{|l|}{ Tukey } \\
\hline & & & \multirow{2}{*}{$\begin{array}{l}\text { Control \& HTN } \\
\text { HTN \& DM } \\
\end{array}$} & \multirow{2}{*}{$\begin{array}{l}\text { Control \& DM } \\
\text { HTN \& HTN - DM }\end{array}$} & \multirow{2}{*}{$\begin{array}{l}\text { Control \& HTN-DM } \\
\text { DM \& HTN - DM }\end{array}$} \\
\hline & $\mathbf{F}$ & P-value & & & \\
\hline \multirow{2}{*}{ Age (Years) } & \multirow{2}{*}{19.083} & \multirow{2}{*}{0.000} & 0.000 & 0.000 & 0.000 \\
\hline & & & 0.917 & 0.591 & 0.927 \\
\hline \multirow{2}{*}{ Weight (Kg) } & \multirow{2}{*}{3.227} & \multirow{2}{*}{0.027} & 0.228 & 0.016 & 0.205 \\
\hline & & & 0.670 & 1.000 & 0.705 \\
\hline \multirow{2}{*}{ BMI } & \multirow{2}{*}{9.851} & \multirow{2}{*}{0.000} & 0.121 & 0.001 & 0.001 \\
\hline & & & 0.031 & 0.280 & 0.719 \\
\hline \multirow{2}{*}{ SBP (mmHg) } & \multirow{2}{*}{23.610} & \multirow{2}{*}{0.000} & 0.000 & 0.012 & 0.000 \\
\hline & & & 0.000 & 0.396 & 0.017 \\
\hline \multirow{2}{*}{ DBP (mmHg) } & \multirow{2}{*}{22.940} & \multirow{2}{*}{0.000} & 0.000 & 0.000 & 0.000 \\
\hline & & & 0.021 & 0.638 & 0.292 \\
\hline \multirow{2}{*}{ Pulse (bpm) } & \multirow{2}{*}{0.416} & \multirow{2}{*}{0.741} & 0.899 & 0.806 & 0.899 \\
\hline & & & 0.698 & 0.899 & 0.899 \\
\hline \multirow{2}{*}{ Total Cholesterol (mg/dl) } & \multirow{2}{*}{3.970} & \multirow{2}{*}{0.011} & 0.021 & 0.032 & 0.042 \\
\hline & & & 0.899 & 0.899 & 0.899 \\
\hline \multirow{2}{*}{$\mathrm{BNP}(\mathrm{pg} / \mathrm{ml})$} & \multirow{2}{*}{275.028} & \multirow{2}{*}{0.000} & 0.000 & 0.000 & 0.000 \\
\hline & & & 0.000 & 0.000 & 0.000 \\
\hline
\end{tabular}

BMI: Body mass index,

SBP: Systolic blood pressure, DBP: Diastolic blood pressure, BNP: Brain Natriuretic Peptide.

$\mathrm{P}$-value $>0.05$ : Insignificant, P-value $<0.05$ : Significant, P-Value $<0.001$ : Highly significant.

Plasma BNP level was the lowest among control subjects and gradually increased to be the highest among hypertensive-diabetic patients as seen from Figure 1. 


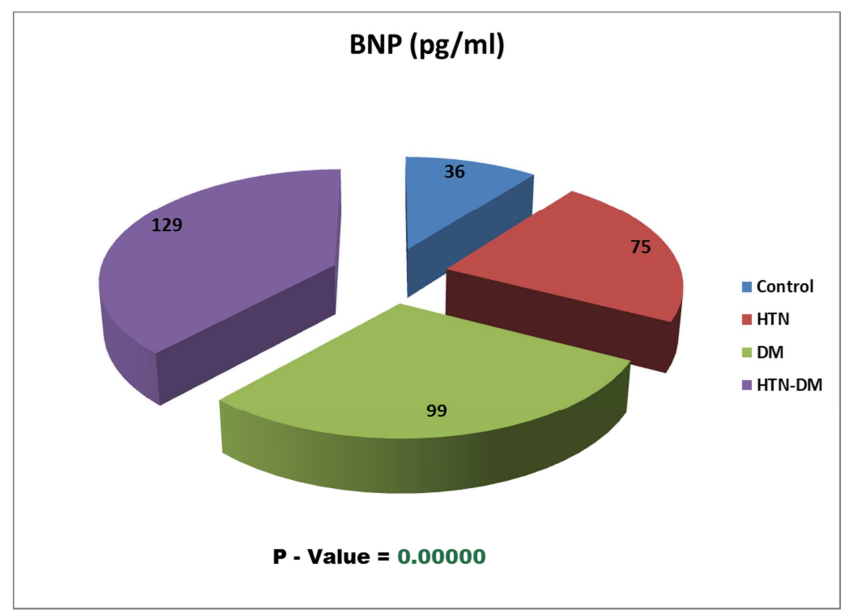

Figure 1. Comparison between study groups regarding BNP levels.

As for the age and genders within each group:

Group 1 included 4 males \& 16 females whose mean age was $32.25 \pm 12.58$.

Group 2 included 11 males \& 9 females, with a mean age of $54.15 \pm 10.06$.

Group 3 included 3 males \& 17 females, whose mean age was $52.05 \pm 7.79$.

Group 4 included 9 males \& 11 females, with a mean age of $50.05 \pm 10.18$.

In all groups, the percentage of smoking persons is less than $5 \%$.

\subsection{Conventional Echocardiography \& Tissue Doppler Imaging}

As shown in Table 2, dimensions LA, AO, IVSd, and PWd were significantly higher among patients groups compared to controls, they were noticeably higher in group 4 (Hypertensive-diabetics). As for mitral Doppler inflow parameters; the E/A ratio was significantly lower among groups 2, 3, and 4 compared to group 1 (controls).

Table 2. Comparison between the study groups regarding Conventional Echocardiographic data and Mitral Annular TDI Parameters.

\begin{tabular}{|c|c|c|c|c|c|}
\hline \multirow{2}{*}{ Items } & & \multicolumn{4}{|l|}{ Group } \\
\hline & & Control $(n=20)$ & HTN $(n=20)$ & DM $(n=20)$ & HTN - DM $(n=20)$ \\
\hline $\mathrm{EF} \%$ & Mean \pm SD & $66.40 \pm 4.49$ & $66.45 \pm 6.61$ & $67.70 \pm 5.12$ & $63.95 \pm 6.75$ \\
\hline $\mathrm{FS} \%$ & Mean \pm SD & $36.50 \pm 2.95$ & $37.05 \pm 5.32$ & $37.40 \pm 4.78$ & $34.45 \pm 4.98$ \\
\hline $\mathrm{LA}(\mathrm{mm})$ & Mean \pm SD & $34.66 \pm 8.29$ & $39.85 \pm 5.52$ & $36.11 \pm 8.36$ & $41.15 \pm 4.66$ \\
\hline $\mathrm{AO}(\mathrm{mm})$ & Mean \pm SD & $27.06 \pm 6.10$ & $31.80 \pm 2.82$ & $28.24 \pm 7.24$ & $32.40 \pm 3.60$ \\
\hline IVSd (cm) & Mean \pm SD & $0.82 \pm 0.12$ & $1.57 \pm 0.25$ & $1.51 \pm 0.21$ & $1.90 \pm 1.93$ \\
\hline LVIDd (cm) & Mean \pm SD & $4.86 \pm 0.36$ & $4.75 \pm 0.53$ & $4.69 \pm 0.46$ & $4.79 \pm 0.51$ \\
\hline LVPWd (cm) & Mean \pm SD & $0.88 \pm 0.10$ & $1.08 \pm 0.20$ & $1.05 \pm 0.16$ & $1.17 \pm 0.17$ \\
\hline $\mathrm{E}(\mathrm{m} / \mathrm{s})$ & Mean \pm SD & $0.80 \pm 0.13$ & $0.60 \pm 0.19$ & $0.58 \pm 0.15$ & $0.66 \pm 0.14$ \\
\hline $\mathrm{A}(\mathrm{m} / \mathrm{s})$ & Mean \pm SD & $0.56 \pm 0.14$ & $0.77 \pm 0.18$ & $0.75 \pm 0.18$ & $0.79 \pm 0.20$ \\
\hline $\mathrm{E} / \mathrm{A}$ ratio & Mean \pm SD & $1.51 \pm 0.49$ & $0.84 \pm 0.40$ & $0.83 \pm 0.37$ & $0.90 \pm 0.36$ \\
\hline$e^{\prime}$ & Mean \pm SD & $0.13 \pm 0.03$ & $0.07 \pm 0.03$ & $0.09 \pm 0.03$ & $0.07 \pm 0.02$ \\
\hline$a^{\prime}$ & Mean \pm SD & $0.08 \pm 0.02$ & $0.11 \pm 0.02$ & $0.11 \pm 0.02$ & $0.12 \pm 0.04$ \\
\hline $\mathrm{E} / \mathrm{e}^{\prime}$ & Mean \pm SD & $6.71 \pm 1.79$ & $9.90 \pm 3.30$ & $7.14 \pm 2.80$ & $9.75 \pm 4.40$ \\
\hline
\end{tabular}

Table 2. Continued.

\begin{tabular}{|c|c|c|c|c|c|}
\hline \multirow{3}{*}{ Items } & \multirow{2}{*}{\multicolumn{2}{|c|}{ ANOVA }} & \multicolumn{3}{|l|}{ Tukey } \\
\hline & & & \multirow{2}{*}{$\begin{array}{l}\text { Control \& HTN } \\
\text { HTN \& DM } \\
\end{array}$} & \multirow{2}{*}{$\begin{array}{l}\text { Control \&DM } \\
\text { HTN \& HTN-DM } \\
\end{array}$} & \multirow{2}{*}{$\begin{array}{l}\text { Control \& HTN-DM } \\
\text { DM \& HTN-DM }\end{array}$} \\
\hline & $\mathbf{F}$ & P-value & & & \\
\hline \multirow{2}{*}{$\mathrm{EF} \%$} & \multirow{2}{*}{1.454} & \multirow{2}{*}{0.234} & 1.000 & 0.894 & 0.547 \\
\hline & & & 0.905 & 0.530 & 0.184 \\
\hline \multirow{2}{*}{$\mathrm{FS} \%$} & \multirow{2}{*}{1.647} & \multirow{2}{*}{0.186} & 0.981 & 0.926 & 0.497 \\
\hline & & & 0.995 & 0.287 & 0.187 \\
\hline \multirow{2}{*}{$\mathrm{LA}(\mathrm{mm})$} & \multirow{2}{*}{3.929} & \multirow{2}{*}{0.012} & 0.050 & 0.909 & 0.020 \\
\hline & & & 0.105 & 0.933 & 0.324 \\
\hline \multirow{2}{*}{ Ao (mm) } & \multirow{2}{*}{4.997} & \multirow{2}{*}{0.003} & 0.028 & 0.893 & 0.010 \\
\hline & & & 0.149 & 0.984 & 0.067 \\
\hline \multirow{2}{*}{ IVSd $(\mathrm{cm})$} & \multirow{2}{*}{4.212} & \multirow{2}{*}{0.008} & 0.536 & 0.496 & 0.293 \\
\hline & & & 0.031 & 0.024 & 0.028 \\
\hline \multirow{2}{*}{ LVIDd (cm) } & \multirow{2}{*}{0.453} & \multirow{2}{*}{0.716} & 0.882 & 0.666 & 0.958 \\
\hline & & & 0.978 & 0.995 & 0.920 \\
\hline \multirow{2}{*}{ LVIDs (cm) } & \multirow{2}{*}{1.397} & \multirow{2}{*}{0.250} & 0.806 & 0.370 & 0.998 \\
\hline & & & 0.886 & 0.709 & 0.283 \\
\hline \multirow{2}{*}{ LVPWd (cm) } & \multirow{2}{*}{10.913} & \multirow{2}{*}{0.000} & 0.054 & 0.615 & 0.003 \\
\hline & & & 0.087 & 0.011 & 1.000 \\
\hline \multirow{2}{*}{$\mathrm{E}(\mathrm{m} / \mathrm{s})$} & \multirow{2}{*}{8.296} & \multirow{2}{*}{0.000} & 0.001 & 0.000 & 0.020 \\
\hline & & & 0.646 & 0.987 & 0.437 \\
\hline \multirow{2}{*}{$\mathrm{A}(\mathrm{m} / \mathrm{s})$} & \multirow{2}{*}{6.834} & 0000 & 0.002 & 0.009 & 0.001 \\
\hline & & 0.000 & 0.994 & 0.963 & 0.880 \\
\hline
\end{tabular}




\begin{tabular}{|c|c|c|c|c|c|}
\hline \multirow{3}{*}{ Items } & \multirow{2}{*}{\multicolumn{2}{|c|}{ ANOVA }} & \multicolumn{3}{|l|}{ Tukey } \\
\hline & & & Control \& HTN & \multirow{2}{*}{$\begin{array}{l}\text { Control \&DM } \\
\text { HTN \& HTN-DM } \\
\end{array}$} & \multirow{2}{*}{$\begin{array}{l}\text { Control \& HTN-DM } \\
\text { DM \& HTN-DM }\end{array}$} \\
\hline & $\mathbf{F}$ & P-value & HTN \& DM & & \\
\hline \multirow{2}{*}{ E/A ratio } & \multirow{2}{*}{12.952} & \multirow{2}{*}{0.000} & 0.000 & 0.000 & 0.000 \\
\hline & & & 0.957 & 1.000 & 0.936 \\
\hline \multirow{2}{*}{$e^{\prime}$} & \multirow{2}{*}{18.312} & \multirow{2}{*}{0.000} & 0.000 & 0.001 & 0.000 \\
\hline & & & 0.031 & 0.743 & 0.281 \\
\hline \multirow{2}{*}{$a^{\prime}$} & \multirow{2}{*}{6.277} & \multirow{2}{*}{0.001} & 0.018 & 0.003 & 0.001 \\
\hline & & & 0.938 & 0.849 & 0.996 \\
\hline \multirow{2}{*}{$\mathrm{E} / \mathrm{e}^{\prime}$} & \multirow{2}{*}{5.493} & \multirow{2}{*}{0.001} & 0.012 & 0.458 & 0.018 \\
\hline & & & 0.040 & 0.238 & 0.974 \\
\hline \multirow{2}{*}{$\mathrm{S}^{\prime}$ wave } & \multirow{2}{*}{1.382} & \multirow{2}{*}{0.255} & 0.190 & 0.636 & 0.636 \\
\hline & & & 0.839 & 0.839 & 1.000 \\
\hline TIME S wave & 1.711 & 0.172 & 0.163 & 0.990 & 0.879 \\
\hline
\end{tabular}

EF\%: Ejection Fraction, FS\%: Fractional Shortening, LA: Left Atrium, AO: Aorta, IVSd: Interventricular septum thickness at end -diastole, LVIDd: Left ventricular internal dimension at end -diastole, LVIDs Left ventricular internal dimension at end -systole, LVPWd: Left ventricular posterior wall thickness at end-diastole, E: Peak velocity of early diastolic transmitral flow, A: Peak velocity of late diastolic transmitral flow.

$\mathrm{e}^{\prime}$ : Peak velocity of mitral annulus at early diastole by pulsed wave Doppler.

$a^{\prime}$ : Peak velocity of mitral annulus at late diastole by pulsed wave Doppler.

$S^{\prime}$ : Velocity of mitral annulus at systole.

$\mathrm{P}$-value $>0.05$ : Insignificant, P-value $<0.05$ : Significant, P-Value $<0.001$ : Highly significant.

Regarding TDI, the peak velocity of the mitral annulus at early diastole by pulsed-wave Doppler ( $\mathrm{e}^{\prime}$ ) showed a significant reduction from group 1 to group 4.

There was an opposite pattern where the values of both peak velocity of the mitral annulus at late diastole by pulsedwave Doppler $\left(a^{\prime}\right)$, and $E / e^{\prime}$ ratio increased from group 1 towards group 4. All of which indicated abnormal LV relaxation as seen from the table.

\subsection{Speckle Tracking Echocardiography}

Table 3 shows a reduction in global LV longitudinal strain (Esys\%) observed from group 1 to group 4. Moreover, the global peak systolic strain rate (SRs ${ }^{s-1}$ ) was significantly lower in group 4 (hypertensive-diabetics) than group 2 (Hypertensives), group 3 (Diabetics), and group 1 (controls) as observed from Table 4.

Table 3. Comparison between the study groups regarding LV peak systolic longitudinal strain Esys\%.

\begin{tabular}{|c|c|c|c|c|c|}
\hline \multirow{2}{*}{ Esys\% } & & \multicolumn{4}{|l|}{ Group } \\
\hline & & Control $(n=20)$ & HTN $(n=20)$ & DM $(n=20)$ & HTN - DM $(n=20)$ \\
\hline Bas. Sep. & Mean \pm SD & $-20.81 \pm 1.53$ & $-15.64 \pm 2.45$ & $-16.13 \pm 3.12$ & $-14.66 \pm 2.04$ \\
\hline Mid. Sep. & Mean \pm SD & $-21.07 \pm 1.76$ & $-15.54 \pm 2.71$ & $-15.95 \pm 3.86$ & $-15.52 \pm 2.67$ \\
\hline Ap. Sep. & Mean \pm SD & $-20.79 \pm 1.45$ & $-15.06 \pm 3.41$ & $-16.25 \pm 3.32$ & $-15.51 \pm 3.14$ \\
\hline Cumulative Sep. & Mean \pm SD & $-20.8 \pm 1.56$ & $-15.4 \pm 2.85$ & $-16.11 \pm 3.39$ & $-15.23 \pm 2.64$ \\
\hline Ap. Lat. & Mean \pm SD & $-20.69 \pm 1.46$ & $-15.48 \pm 2.78$ & $-15.63 \pm 3.74$ & $-14.51 \pm 2.57$ \\
\hline Mid. Lat. & Mean \pm SD & $-20.81 \pm 1.54$ & $-15.24 \pm 3.45$ & $-15.56 \pm 3.27$ & $-13.59 \pm 3.43$ \\
\hline Bas. Lat. & Mean \pm SD & $-21.24 \pm 1.92$ & $-14.74 \pm 3.53$ & $-15.67 \pm 2.78$ & $-14.80 \pm 3.04$ \\
\hline Cumulative Lat. & Mean \pm SD & $-20.91 \pm 1.64$ & $-15.15 \pm 3.23$ & $-15.62 \pm 3.23$ & $-14.30 \pm 3.03$ \\
\hline Bas. Inf. & Mean \pm SD & $-21.83 \pm 2.03$ & $-15.43 \pm 3.39$ & $-15.68 \pm 3.19$ & $-15.18 \pm 3.23$ \\
\hline Mid. Inf. & Mean \pm SD & $-20.88 \pm 1.41$ & $-15.12 \pm 3.56$ & $-16.09 \pm 3.87$ & $-15.75 \pm 3.67$ \\
\hline Ap. Inf. & Mean \pm SD & $-21.23 \pm 1.50$ & $-16.05 \pm 2.93$ & $-15.68 \pm 3.20$ & $-14.57 \pm 3.67$ \\
\hline Cumulative Inf. & Mean \pm SD & $-21.31 \pm 1.69$ & $-15.53 \pm 3.27$ & $-15.82 \pm 3.38$ & $-15.17 \pm 3.50$ \\
\hline Ap. Ant. & Mean \pm SD & $-21.00 \pm 1.31$ & $-15.17 \pm 3.47$ & $-16.58 \pm 3.94$ & $-14.19 \pm 4.36$ \\
\hline Mid. Ant. & Mean \pm SD & $-21.35 \pm 1.49$ & $-14.36 \pm 3.51$ & $-15.75 \pm 3.73$ & $-15.47 \pm 3.76$ \\
\hline Bas. Ant. & Mean \pm SD & $-20.80 \pm 1.78$ & $-15.92 \pm 3.30$ & $-16.76 \pm 3.87$ & $-14.80 \pm 3.95$ \\
\hline Cumulative Ant. & Mean \pm SD & $-21.05 \pm 1.53$ & $-15.15 \pm 3.43$ & $-16.36 \pm 3.81$ & $-14.83 \pm 4.00$ \\
\hline Bas. Post. & Mean \pm SD & $-21.07 \pm 1.58$ & $-15.33 \pm 3.09$ & $-15.63 \pm 2.35$ & $-15.07 \pm 2.99$ \\
\hline Mid. Post. & Mean \pm SD & $-20.87 \pm 1.52$ & $-15.98 \pm 2.45$ & $-16.13 \pm 2.84$ & $-14.75 \pm 3.15$ \\
\hline Ap. Post. & Mean \pm SD & $-20.83 \pm 2.10$ & $-15.69 \pm 3.67$ & $-15.73 \pm 3.88$ & $-14.62 \pm 3.60$ \\
\hline Cumulative Post. & $\mathrm{Mean} \pm \mathrm{SD}$ & $-20.93 \pm 1.73$ & $-15.67 \pm 3.07$ & $-15.83 \pm 3.04$ & $-14.81 \pm 3.21$ \\
\hline Ap. Ant. Sep. & Mean \pm SD & $-20.59 \pm 1.72$ & $-16.14 \pm 2.94$ & $-16.16 \pm 2.49$ & $-15.51 \pm 3.37$ \\
\hline Mid. Ant. Sep. & Mean \pm SD & $-20.64 \pm 1.57$ & $-15.84 \pm 3.49$ & $-16.04 \pm 2.41$ & $-15.55 \pm 4.03$ \\
\hline Bas. Ant. Sep. & Mean \pm SD & $-20.85 \pm 1.33$ & $-15.85 \pm 3.17$ & $-16.21 \pm 2.79$ & $-15.33 \pm 3.67$ \\
\hline Cumulative Ant. Sep. & Mean \pm SD & $-20.69 \pm 1.52$ & $-15.94 \pm 3.16$ & $-16.14 \pm 2.53$ & $-15.46 \pm 3.64$ \\
\hline Cumulative Global LV Esys\% & Mean \pm SD & $-20.97 \pm 1.61$ & $-15.48 \pm 3.16$ & $-15.98 \pm 3.24$ & $-14.97 \pm 3.36$ \\
\hline
\end{tabular}


Table 3. Continued.

\begin{tabular}{|c|c|c|c|c|c|}
\hline \multirow{3}{*}{ Esys\% } & \multirow{2}{*}{\multicolumn{2}{|c|}{ ANOVA }} & \multicolumn{3}{|l|}{ Tukey } \\
\hline & & & Control \& HTN & Control \& DM & Control \& HTN-DM \\
\hline & $\mathbf{F}$ & P-value & HTN \& DM & HTN \& HTN-DM & DM \& HTN-DM \\
\hline \multirow{2}{*}{ Bas. Sep. } & \multirow{2}{*}{26.867} & \multirow{2}{*}{0.000} & 0.000 & 0.000 & 0.000 \\
\hline & & & 0.915 & 0.552 & 0.208 \\
\hline \multirow{2}{*}{ Mid. Sep. } & \multirow{2}{*}{18.098} & \multirow{2}{*}{0.000} & 0.000 & 0.000 & 0.000 \\
\hline & & & 0.968 & 1.000 & 0.963 \\
\hline \multirow{2}{*}{ Ap. Sep. } & \multirow{2}{*}{16.095} & \multirow{2}{*}{0.000} & 0.000 & 0.000 & 0.000 \\
\hline & & & 0.582 & 0.963 & 0.858 \\
\hline \multirow{2}{*}{ Cumulative Sep. } & \multirow{2}{*}{59.394} & \multirow{2}{*}{0.000} & 0.001 & 0.001 & 0.001 \\
\hline & & & 0.091 & 0.027 & 0.119 \\
\hline \multirow{2}{*}{ Ap. Lat. } & \multirow{2}{*}{20.388} & \multirow{2}{*}{0.000} & 0.000 & 0.000 & 0.000 \\
\hline & & & 0.998 & 0.682 & 0.575 \\
\hline \multirow{2}{*}{ Mid. Lat. } & \multirow{2}{*}{21.306} & & 0.000 & 0.000 & 0.000 \\
\hline & & 0.000 & 0.986 & 0.320 & 0.175 \\
\hline & & & 0.000 & 0.000 & 0.000 \\
\hline Bas. Lat. & 23.451 & 0.000 & 0.741 & 1.000 & 0.775 \\
\hline & & & 0.001 & 0.001 & 0.001 \\
\hline Cumulatıve Lat. & 65.771 & 0.000 & 0.054 & 0.112 & 0.173 \\
\hline & & & 0.000 & 0.000 & 0.000 \\
\hline Bas. Int. & 22.737 & 0.000 & 0.993 & 0.993 & 0.951 \\
\hline Mid Inf & 12973 & 0.000 & 0.000 & 0.000 & 0.000 \\
\hline Mnd. iml. & 12.913 & & 0.786 & 0.928 & 0.988 \\
\hline An Inf & 20377 & 0000 & 0.000 & 0.000 & 0.000 \\
\hline Ар. пा1. & 20.311 & 0.000 & 0.979 & 0.391 & 0.633 \\
\hline Cumulative Inf & 54.808 & 0000 & 0.001 & 0.001 & 0.001 \\
\hline Cumulative int. & 54.808 & 0.000 & 0.035 & 0.044 & 0.077 \\
\hline An Ant & 15002 & 0000 & 0.000 & 0.001 & 0.000 \\
\hline & 15.002 & 0.000 & 0.581 & 0.806 & 0.140 \\
\hline & & & 0.000 & 0.000 & 0.000 \\
\hline Mid. Ant. & 18.488 & 0.000 & 0.538 & 0.707 & 0.993 \\
\hline & 12239 & & 0.000 & 0.001 & 0.000 \\
\hline Bas. Ant. & 12.239 & 0.000 & 0.857 & 0.713 & 0.255 \\
\hline & & & 0.001 & 0.001 & 0.001 \\
\hline Cumulatıve Ant. & 44.686 & 0.000 & 0.137 & 0.036 & 0.162 \\
\hline & 24044 & 0000 & 0.000 & 0.000 & 0.000 \\
\hline Bas. Post. & 24.944 & 0.000 & 0.983 & 0.989 & 0.902 \\
\hline Mid Post & 22099 & 0000 & 0.000 & 0.000 & 0.000 \\
\hline Mira. Post. & 22.099 & 0.000 & 0.998 & 0.434 & 0.331 \\
\hline An Post & 13567 & 0000 & 0.000 & 0.000 & 0.000 \\
\hline Ap. Post. & $13.56 /$ & 0.000 & 1.000 & 0.753 & 0.728 \\
\hline Cumulative Post & 58050 & 0000 & 0.001 & 0.001 & 0.001 \\
\hline Cumulative rost. & & & 0.022 & 0.111 & 0.133 \\
\hline An. Ant. Sen & 15101 & 0000 & 0.000 & 0.000 & 0.000 \\
\hline & & & 1.000 & 0.883 & 0.871 \\
\hline Mid. Ant. Sep. & 12.814 & 0000 & 0.000 & 0.000 & 0.000 \\
\hline & 12.814 & 0.000 & 0.997 & 0.990 & 0.956 \\
\hline & 15773 & & 0.000 & 0.000 & 0.000 \\
\hline Bas. Ant. Sep. & $15.7 / 3$ & 0.000 & 0.979 & 0.940 & 0.769 \\
\hline & & & 0.001 & 0.001 & 0.001 \\
\hline Cumulative Ant. Sep. & 44.787 & 0.000 & 0.027 & 0.052 & 0.088 \\
\hline Cumulative Global LV & 322657 & 0000 & 0.001 & 0.001 & 0.001 \\
\hline Esys\% & $322.03 /$ & 0.000 & 0.153 & 0.140 & 0.001 \\
\hline
\end{tabular}

Ap.: Apical Segment, Bas.: Basal Segment, Sep.: Septal wall, Lat.: Lateral wall, Inf.: Inferior wall, Ant.: Anterior wall, Post.: Posterior wall, Ant. Sep.: Antero-Septal wall.

P-value $>0.05$ : Insignificant, P-value $<0.05$ : Significant, P-Value $<0.001$ : Highly significant.

Table 4. Comparison between the study groups regarding LV Strain Rate at Peak Systole $\left(S R s^{S-1}\right)$.

\begin{tabular}{lllll}
\hline \multirow{2}{*}{ SRs ${ }^{\text {S-1 }}$} & & Group & & DM \\
\cline { 2 - 5 } & & Control & HTN & $-1.05 \pm 0.03$ \\
Bas. Sep. & Mean \pm SD & $-1.01 \pm 0.49$ & $-0.94 \pm 0.07$ & $-0.91 \pm 0.08$ \\
Mid. Sep. & Mean \pm SD & $-1.09 \pm 0.06$ & $-0.94 \pm 0.09$ & $-1.05 \pm 0.02$ \\
Ap. Sep. & Mean \pm SD & $-0.91 \pm 0.55$ & $-0.94 \pm 0.10$ & $-0.84 \pm 0.64$ \\
Cumulative Sep. & Mean \pm SD & $-1.02 \pm 0.40$ & $-0.94 \pm 0.08$ & $-0.98 \pm 0.38$ \\
Ap. Lat. & Mean \pm SD & $-1.14 \pm 0.10$ & $-0.83 \pm 0.43$ & $-1.05 \pm 0.04$ \\
\hline
\end{tabular}




\begin{tabular}{|c|c|c|c|c|c|}
\hline \multirow{2}{*}{ SRs ${ }^{\text {S-1 }}$} & & \multicolumn{4}{|l|}{ Group } \\
\hline & & Control & HTN & DM & HTN - DM \\
\hline Mid. Lat. & Mean \pm SD & $-1.11 \pm 0.06$ & $-0.91 \pm 0.08$ & $-1.06 \pm 0.03$ & $-0.91 \pm 0.07$ \\
\hline Bas. Lat. & Mean \pm SD & $-1.12 \pm 0.06$ & $-0.84 \pm 0.41$ & $-1.06 \pm 0.03$ & $-0.88 \pm 0.07$ \\
\hline Cumulative Lat. & Mean \pm SD & $-1.12 \pm 0.08$ & $-0.86 \pm 0.34$ & $-1.06 \pm 0.03$ & $-0.88 \pm 0.08$ \\
\hline Bas. Inf. & Mean \pm SD & $-1.10 \pm 0.05$ & $-0.88 \pm 0.10$ & $-1.05 \pm 0.04$ & $-0.87 \pm 0.05$ \\
\hline Mid. Inf. & Mean \pm SD & $-1.13 \pm 0.09$ & $-0.91 \pm 0.09$ & $-0.96 \pm 0.46$ & $-0.91 \pm 0.07$ \\
\hline Ap. Inf. & Mean \pm SD & $-1.09 \pm 0.05$ & $-0.91 \pm 0.12$ & $-1.06 \pm 0.03$ & $-0.91 \pm 0.08$ \\
\hline Ap. Ant. & Mean \pm SD & $-1.10 \pm 0.06$ & $-0.89 \pm 0.09$ & $-1.04 \pm 0.02$ & $-0.75 \pm 0.63$ \\
\hline Mid. Ant. & Mean \pm SD & $-1.11 \pm 0.06$ & $-0.92 \pm 0.12$ & $-1.06 \pm 0.03$ & $-0.89 \pm 0.09$ \\
\hline Bas. Ant & Mean \pm SD & $-1.10 \pm 0.06$ & $-0.94 \pm 0.08$ & $-1.06 \pm 0.03$ & $-0.87 \pm 0.08$ \\
\hline Cumulative Ant. & Mean \pm SD & $-1.10 \pm 0.06$ & $-0.92 \pm 0.10$ & $-1.05 \pm 0.03$ & $-0.84 \pm 0.37$ \\
\hline Bas. Post. & Mean \pm SD & $-1.14 \pm 0.20$ & $-0.89 \pm 0.10$ & $-1.05 \pm 0.03$ & $-0.88 \pm 0.08$ \\
\hline Mid. Post. & Mean \pm SD & $-1.08 \pm 0.05$ & $-0.91 \pm 0.10$ & $-1.06 \pm 0.03$ & $-0.87 \pm 0.09$ \\
\hline Ap. Post. & Mean \pm SD & $-1.09 \pm 0.06$ & $-0.91 \pm 0.10$ & $-1.05 \pm 0.02$ & $-0.92 \pm 0.08$ \\
\hline Ap. Ant. Sep. & Mean \pm SD & $-1.13 \pm 0.15$ & $-0.90 \pm 0.11$ & $-1.05 \pm 0.03$ & $-0.87 \pm 0.09$ \\
\hline Mid. Ant. Sep. & Mean \pm SD & $-1.15 \pm 0.09$ & $-0.93 \pm 0.11$ & $-1.05 \pm 0.03$ & $-0.92 \pm 0.07$ \\
\hline Bas. Ant. Sep. & Mean \pm SD & $-1.09 \pm 0.05$ & $-0.90 \pm 0.10$ & $-0.95 \pm 0.46$ & $-0.89 \pm 0.10$ \\
\hline Cumulative Ant. Sep. & Mean \pm SD & $-1.12 \pm 0.11$ & $-0.91 \pm 0.10$ & $-1.02 \pm 0.27$ & $-0.90 \pm 0.09$ \\
\hline Cumulative Global LV SRs s ${ }^{-1}$. & Mean \pm SD & $-1.10 \pm 0.19$ & $-0.90 \pm 0.17$ & $-1.03 \pm 0.22$ & $-0.88 \pm 0.17$ \\
\hline
\end{tabular}

Table 4. Continued.

\begin{tabular}{|c|c|c|c|c|c|}
\hline \multirow{3}{*}{ SRs ${ }^{\mathrm{s}-1}$} & \multirow{2}{*}{\multicolumn{2}{|c|}{ ANOVA }} & \multicolumn{3}{|l|}{ Tukey } \\
\hline & & & Control \& HTN & Control \& DM & Control \& HTN - DM \\
\hline & $\mathbf{F}$ & P-value & HTN \& DM & HTN \& HTN - DM & DM \& HTN - DM \\
\hline \multirow{2}{*}{ Bas. Sep. } & \multirow{2}{*}{1.290} & \multirow{2}{*}{0.284} & 0.849 & 0.953 & 0.587 \\
\hline & & & 0.543 & 0.968 & 0.285 \\
\hline \multirow{2}{*}{ Mid. Sep. } & \multirow{2}{*}{43.617} & \multirow{2}{*}{0.000} & 0.000 & 0.257 & 0.000 \\
\hline & & & 0.000 & 0.094 & 0.000 \\
\hline \multirow{2}{*}{ Ap. Sep. } & \multirow{2}{*}{0.215} & \multirow{2}{*}{0.886} & 0.997 & 0.944 & 1.000 \\
\hline & & & 0.874 & 0.998 & 0.937 \\
\hline \multirow{2}{*}{ Cumulative Sep. } & \multirow{2}{*}{1.998} & \multirow{2}{*}{0.114} & 0.368 & 0.817 & 0.097 \\
\hline & & & 0.842 & 0.896 & 0.450 \\
\hline \multirow{2}{*}{ Ap. Lat. } & \multirow{2}{*}{8.508} & \multirow{2}{*}{0.000} & 0.000 & 0.652 & 0.002 \\
\hline & & & 0.014 & 0.968 & 0.047 \\
\hline \multirow{2}{*}{ Mid. Lat. } & \multirow{2}{*}{50.967} & \multirow{2}{*}{0.000} & 0.000 & 0.061 & 0.000 \\
\hline & & & 0.000 & 0.999 & 0.000 \\
\hline \multirow{2}{*}{ Bas. Lat. } & \multirow{2}{*}{8.178} & \multirow{2}{*}{0.000} & 0.000 & 0.828 & 0.004 \\
\hline & & & 0.008 & 0.924 & 0.044 \\
\hline \multirow{2}{*}{ Cumulative Lat. } & \multirow{2}{*}{29.848} & & 0.001 & 0.059 & 0.001 \\
\hline & & 0.000 & 0.001 & 0.899 & 0.001 \\
\hline & & & 0.000 & 0.045 & 0.000 \\
\hline Bas. Inf. & 68.082 & 0.000 & 0.000 & 0.959 & 0.000 \\
\hline & & & 0.026 & 0.130 & 0.030 \\
\hline Mid. Int. & 3.664 & 0.016 & 0.908 & 1.000 & 0.926 \\
\hline & & & 0.000 & 0.706 & 0.000 \\
\hline Ap. Inf. & 31.288 & 0.000 & 0.000 & 0.999 & 0.000 \\
\hline & & & 0.001 & 0.016 & 0.001 \\
\hline Cumulative Inf. & 27.031 & 0.000 & 0.001 & 0.899 & 0.001 \\
\hline & & & 0.177 & 0.921 & 0.005 \\
\hline Ap. Ant. & 4.737 & 0.004 & 0.489 & 0.507 & 0.031 \\
\hline & & & 0.000 & 0.202 & 0.000 \\
\hline Mid. Ant. & 35.581 & 0.000 & 0.000 & 0.646 & 0.000 \\
\hline & & & 0.000 & 0.343 & 0.000 \\
\hline Bas. Ant & 50.760 & 0.000 & 0.000 & 0.004 & 0.000 \\
\hline & & & 0.001 & 0.491 & 0.001 \\
\hline Cumulative Ant. & 24.158 & 0.000 & 0.001 & 0.098 & 0.001 \\
\hline & & & 0.000 & 0.110 & 0.000 \\
\hline Bas. Post. & 21.467 & 0.000 & 0.001 & 0.990 & 0.000 \\
\hline & & & 0.000 & 0.802 & 0.000 \\
\hline Mid. Post. & 39.028 & 0.000 & 0.000 & 0.266 & 0.000 \\
\hline Ap. Post. & 35.268 & 0.000 & 0.000 & 0.150 & 0.000 \\
\hline
\end{tabular}




\begin{tabular}{|c|c|c|c|c|c|}
\hline \multirow{3}{*}{ SRs ${ }^{\text {S-1 }}$} & \multirow{2}{*}{\multicolumn{2}{|c|}{ ANOVA }} & \multicolumn{3}{|l|}{ Tukey } \\
\hline & & & Control \& HTN & Control \& DM & Control \& HTN - DM \\
\hline & $\mathbf{F}$ & P-value & HTN \& DM & HTN \& HTN - DM & DM \& HTN - DM \\
\hline \multirow{3}{*}{ Cumulative Post. } & \multirow{3}{*}{82.209} & \multirow{3}{*}{0.000} & 0.000 & 0.970 & 0.000 \\
\hline & & & 0.001 & 0.001 & 0.001 \\
\hline & & & 0.001 & 0.795 & 0.001 \\
\hline \multirow{2}{*}{ Ap. Ant. Sep. } & \multirow{2}{*}{28.760} & \multirow{2}{*}{0.000} & 0.000 & 0.044 & 0.000 \\
\hline & & & 0.000 & 0.841 & 0.000 \\
\hline \multirow{2}{*}{ Mid. Ant. Sep. } & \multirow{2}{*}{35.196} & \multirow{2}{*}{0.000} & 0.000 & 0.002 & 0.000 \\
\hline & & & 0.000 & 0.997 & 0.000 \\
\hline \multirow{2}{*}{ Bas. Ant. Sep. } & \multirow{2}{*}{2.834} & \multirow{2}{*}{0.044} & 0.073 & 0.286 & 0.053 \\
\hline & & & 0.905 & 1.000 & 0.863 \\
\hline \multirow{2}{*}{ Cumulative Ant. Sep. } & \multirow{2}{*}{26.770} & \multirow{2}{*}{0.000} & 0.001 & 0.001 & 0.001 \\
\hline & & & 0.001 & 0.899 & 0.001 \\
\hline Cumulative Global LV SRs s ${ }^{-1}$. & 111.799 & 0.000 & 0.001 & 0.001 & 0.001 \\
\hline
\end{tabular}

Ap.: Apical Segment, Bas.: Basal Segment, Sep.: Septal wall, Lat.: Lateral wall, Inf.: Inferior wall, Ant.: Anterior wall, Post.: Posterior wall, Ant. Sep.: Antero-Septal wall.

P-value $>0.05$ : Insignificant, P-value $<0.05$ : Significant, P-Value $<0.001$ : Highly significant.

The results also show a significant reduction of global early diastolic SR, from group 1 towards group 4. In contrast, global late diastolic SR was significantly higher in group 2 (Hypertensives) and group 4 (Hypertensive-diabetics) compared to group 3 (Diabetics). It had the lowest values among group 1 (Controls).

\subsection{Correlations}

The study showed a significant positive correlation between the mean value of plasma BNP levels and global systolic strain in group 4 (Hypertensive-diabetics) $(\mathrm{r}=0.25$, $\mathrm{P}$ value $=0.000$ ). This can be clearly seen in Figures 2 and 3 . ROC curves, presented in Figure 4, were constructed to explore the optimum cutoff points for strain and strain rate that separated patients from controls.

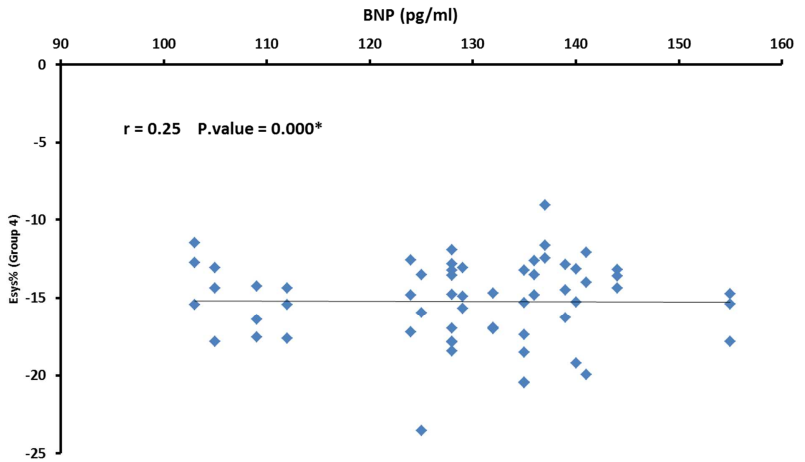

Figure 2. Correlation between BNP level and global peak systolic strain (Esys \%) in hypertensive diabetic patients.

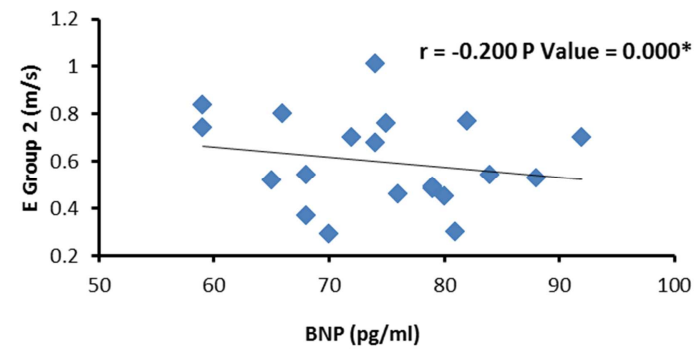

a. (E) in hypertensive patients.

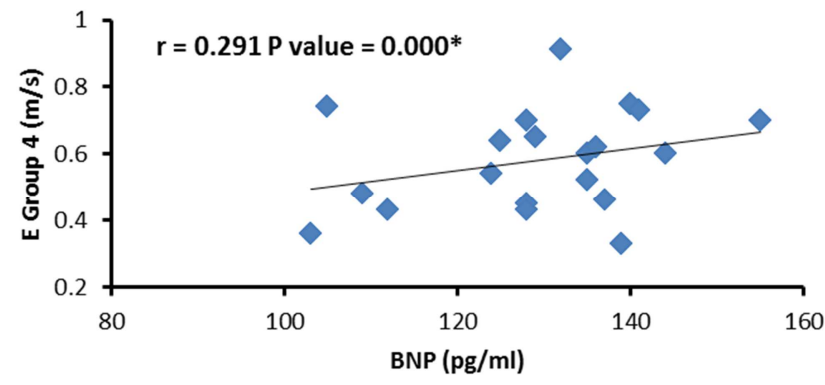

b. (E) in hypertensive diabetic patients.

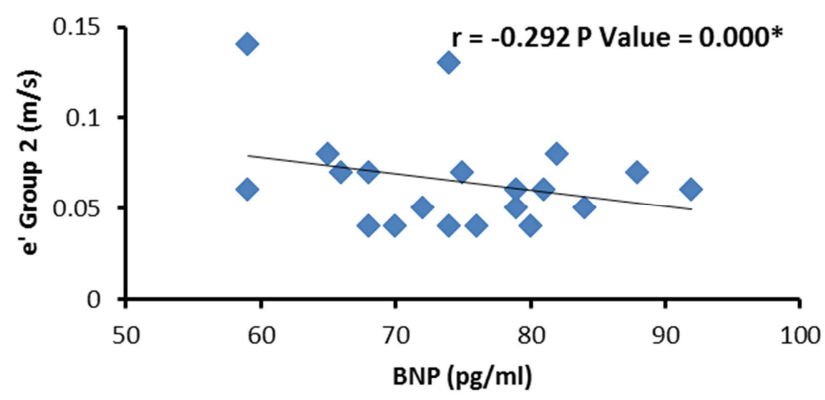

c. $\left(\mathrm{e}^{\prime}\right)$ in hypertensive patients.

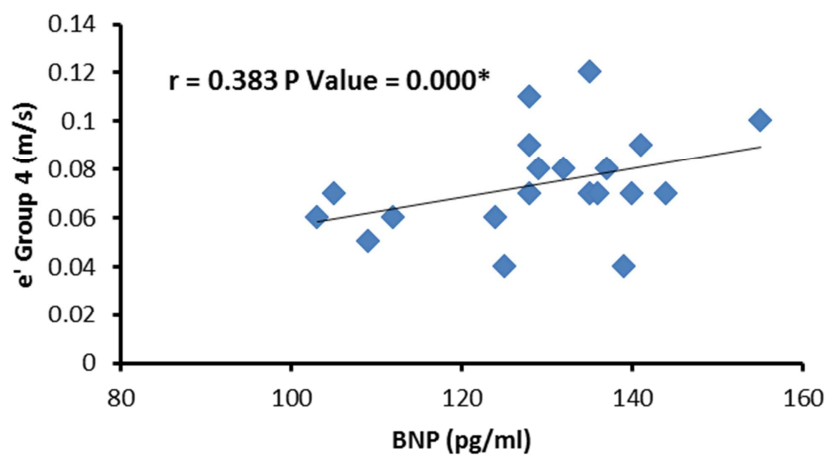

d. $\left(e^{\prime}\right)$ in hypertensive diabetic patients.

Figure 3. Correlation between BNP level and peak velocity of early diastolic transmitral flow $(E)$, and peak velocity of mitral annulus at early diastole by pulsed-wave Doppler ( $\left.e^{\prime}\right)$. 
Esys\%

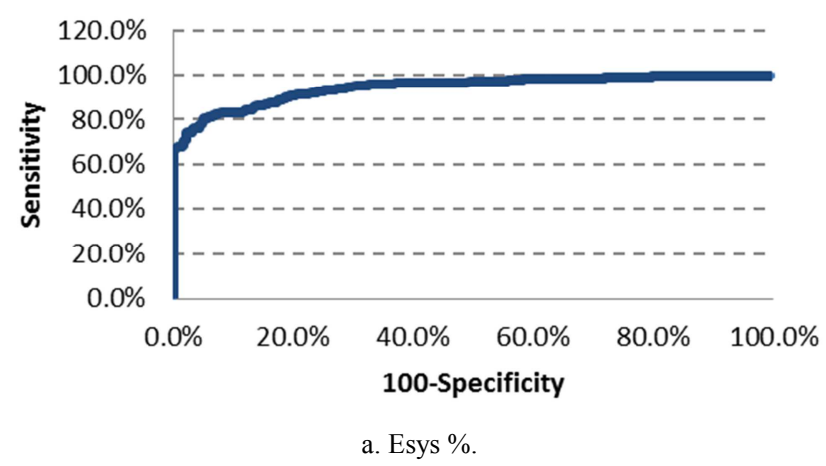

SRs s-1

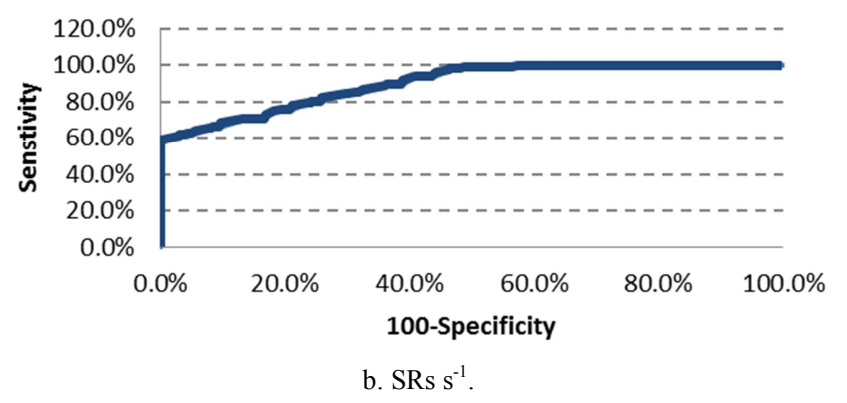

SRe s-1

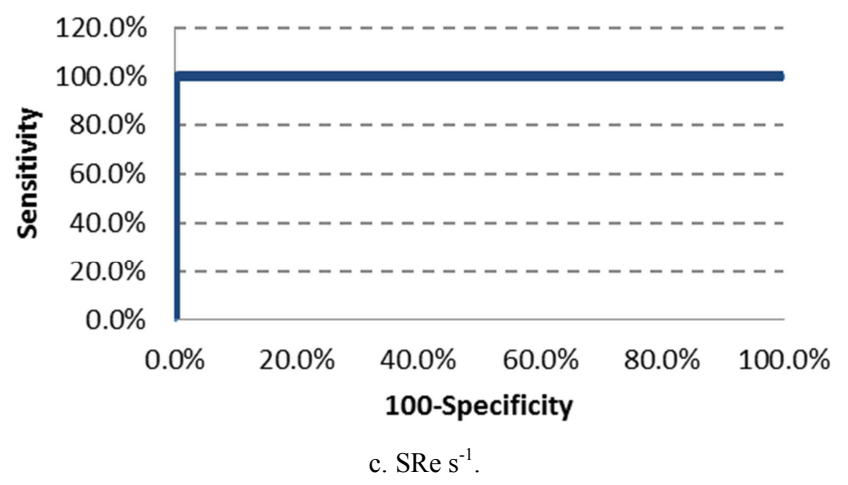

SRa s-1

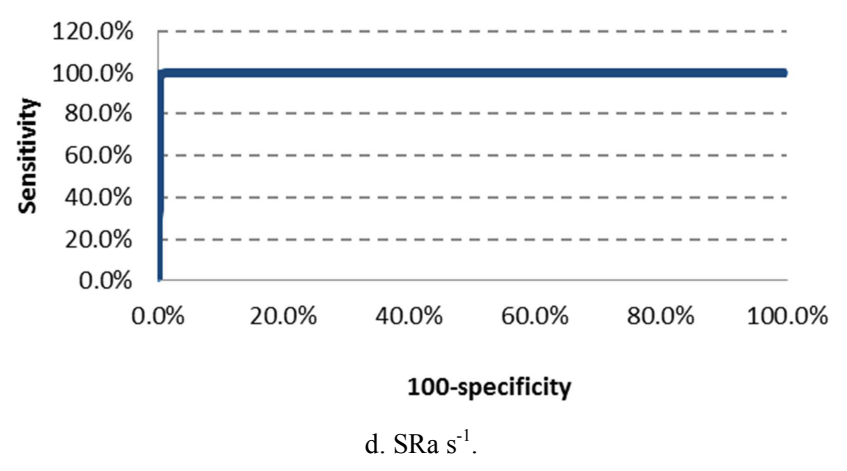

Figure 4. ROC curve between patients and controls.

\section{Discussion}

Conventional echocardiographic parameters have been developed over the years to provide an estimate of LV systolic function. Among them is LVEF, which is the most clinically relevant parameter for this purpose. However, LVEF has several limitations. First, it does not measure the myocardial contractile function directly. Second, it is influenced by factors such as loading conditions, heart rate, etc. Third, and the most important, it is not sensitive enough to detect subtle changes in the contractile function and therefore is not suitable for detecting subclinical myocardial damage which may have major therapeutic and prognostic implications in a variety of clinical conditions.

Using speckle tracking echocardiography, strain imaging offers a means to directly quantify the extent of myocardial contraction attempting to overcome many of the limitations of LVEF. The most important role of STE is to provide a quantitative, objective measure of LV systolic function which can accurately detect subtle changes in the myocardial function. Global Longitudinal Strain (GLS) appears to be the most suited of all myocardial deformation parameters for this purpose. It has better reproducibility and is much more sensitive to the subtle changes that occur, indicating early myocardial damage.

LV systolic function assessed by conventional echocardiography in this study showed no significant difference between patient groups and the control group regarding $\mathrm{LV}$ ejection fraction (EF). These findings were in concordance with the findings from Wang et al [25] who studied 90 patients with diabetes, 36 of which had co-existent hypertension, and categorized them into 3 groups; control, patients with diabetes, and patients with both diabetes and hypertension. The study revealed that, concerning cardiac function assessed by conventional echocardiography, the three groups showed similar LVEF values.

Similar results were also recorded by Bakhoum et al [26] who enrolled 60 diabetic patients and 30 controls in the study and noticed that there was no statistically significant difference between patients and control subjects regarding LV ejection fraction (EF).

In this study, the highly significant reduction of LV systolic strain and strain rate values, from the control group towards the hypertensive group, diabetic group, to become the least for the hypertensive diabetic group, despite the similar LVEF values, supports that STE is superior to conventional echocardiography in detecting the early impairment of LV systolic function. These findings were supported by Stevanovic \& Deklava [27] who studied 121 diabetic patients and 41 healthy subjects and revealed that Global longitudinal strain (GLS) was found to be significantly lower in diabetic patients compared to the controls, despite the normal LVEF.

Our results are also in consonance with Soufi-Taleb \& Meziane Tani [28] who studied 30 hypertensive patients with diabetes and 30 non-diabetic hypertensive patients. Their results showed that both patient categories had LVEF greater than $55 \%$ by conventional echocardiography, but calculating GLS showed lower rates of GLS in 18 diabetic hypertensive patients against 9 nondiabetic hypertensive patients. 
Zhang et al [29] who studied diabetic patients and subdivided them into diabetics with good glycemic control \& diabetics with poor glycemic control, found out that despite similar LVEF, the global longitudinal strain was reduced in both diabetic groups compared to the control group.

As per Ikonomidis et al [30] who studied 320 hypertensive patients and 160 controls, Global longitudinal strain and systolic strain rate were impaired in hypertensive patients compared with controls ( $\mathrm{P}$-Value $<0.05$ ).

Findings from this study were further supported by Wang et al [25] who pointed out that GLS was significantly lower in patients with diabetes only than in controls, the study also highlighted that the patients with diabetes and hypertension showed significantly lower GLS than both controls as well as patients with diabetes only.

Yu Kolesnyk [31] who enrolled 4 patient groups into his study, dividing them into group 1 which included normoglycemic hypertensive patients without insulin resistance (IR), group 2 which included normoglycemic hypertensive patients with IR, group 3 which included hypertensive patients with impaired fasting glucose, and group 4 which included hypertensive patients with diabetes. The findings of the study showed that global longitudinal strain was significantly reduced in group 4 (patients with diabetes and hypertension) compared with other patients, which also supports the findings from the present study.

Ernande et al [32] also pointed out that 36 out of 154 diabetic patients enrolled in the study, who had preserved EF, did show LV systolic dysfunction, defined by the reduced GLS below the cut-off point set by the observers.

This is justified by the fact that the spatial organization of myocardial fibers has a major impact on cardiac mechanics. Among the three layers of the myocardium, longitudinal subendocardial fibers are most susceptible to the adverse effects of ischemia, hypo-perfusion, and age-related interstitial fibrosis. Thus, early manifestations of myocardial disorders are characterized by impaired longitudinal function. And it is the first to decline in hypertensive diseases, as per Ikonomidis et al [30], Yu. Kolesnyk [31], and Yau-Huei Lai et al [33].

STE allows objective quantification of cardiac motion and deformation, irrespective of echo beam direction, thus providing a detailed understanding of cardiac mechanics from all aspects. Therefore, evaluating longitudinal function may serve as an early marker, by accomplishing global longitudinal strain measurement at an early stage of myocardial damage before the overt development of chamber-level failure, such as reduced EF. Moreover, the limited sensitivity of EF is attributed to being measured in the parasternal long-axis view, so it is not affected by the reduction of longitudinal strain, as per Imbalzano, et al [34].

These studies, alongside the present study, confirm that LVEF is well established as not being the most sensitive index for the detection of subclinical LV systolic function impairment. Thereby, the evaluation of LV systolic strain and strain rate is crucial for detecting subclinical myocardial deformation and the reduction of intrinsic myocardial contractility at its early stages, especially in hypertensive and diabetic patients.

Evaluating LV diastolic function with conventional echocardiography and TDI:

This study demonstrated a reduction in peak velocity of early diastolic transmitral flow (E), elevation in peak velocity of late diastolic transmitral flow (A), and inversion in E/A ratio in hypertensive patients, diabetic patients, and hypertensive diabetic patients compared to the controls, indicating impairment in LV diastolic function, which was more evident in hypertensive diabetic patients. The study also revealed a reduction in global peak velocity of the mitral annulus at early diastole $\left(\mathrm{e}^{\prime}\right)$ and elevation of $\mathrm{E} / \mathrm{e}^{\prime}$ ratio which was significantly different when the 3 patient groups were compared to controls, these TDI parameters are also indicators of diastolic dysfunction.

Consistent with these previous data are findings from Li et al [35], who studied 3 groups of patients; control group, hypertensive group, hypertensive diabetics groups, and stated that the hypertensive group had lower E wave and higher A wave than the control group, while the hypertensive diabetic group had lower E wave and higher A wave than both control $\&$ hypertensive group.

Diabetic patients from the study of Bakhoum et al [26] also had a significantly higher peak $\mathrm{A}$ wave and $\mathrm{E} / \mathrm{e}^{\prime}$ ratio compared to controls. Though, the same study showed dissimilarity regarding peak E velocity as well as E/A ratio, where they found no significant difference between diabetic patients and controls. Wang et al [25] showed similar results regarding the $\mathrm{E} / \mathrm{e}^{\prime}$ ratio, which was significantly higher among hypertensive diabetic patients compared to controls. Yet, there was some disparity regarding E/A ratio, where patients and controls had similar E/A ratios. Ernande et al [36] who compared diabetic patients to healthy controls revealed lower $\mathrm{E} / \mathrm{A}$ ratio and $\mathrm{e}^{\prime}$ wave and higher $\mathrm{E} / \mathrm{e}^{\prime}$ ratio among diabetics, which are similar to the findings from this study.

This impaired diastolic function is attributed to the fact that the subendocardial layer is affected first due to ventricular remodeling and subendocardial ischemia due to micro-vascular affection $[37,38]$, which eventually leads to LV thickening, myocardial fibrosis, and LV stiffness as the disease progresses. [39]

Diastolic Strain rate:

Findings from this study exhibited a significant reduction in early diastolic strain rate values in all the 3 patient groups compared to controls, being the least among hypertensive diabetic patients. The late diastolic strain rate also showed a significant difference between the patients and controls. These findings confirm the impairment of diastolic function among the patient groups.

Early diastolic strain rate findings were compatible with the findings from Hamed et al [40] which stated that early diastolic strain rate was significantly reduced in hypertensive patients compared to controls.

Yu Kolesnyk [31] also stated that patients with hypertension and diabetes had a significantly lower longitudinal strain rate at early diastole, compared to other 
groups included in the study.

Findings from this study validate what previous studies stated; early subclinical changes in cardiac function could be detected by TDI or 2DSTE. However, TDI is angledependent and only provides velocity and strain information in the long axis direction [41-43]. 2DSTE overcomes the angle dependency of TDI, yet it is confined to a twodimensional plane and therefore ignores the complex motion in the third dimension, which causes the loss of acoustic speckles and less accurate results [44].

GLS has been shown to be a powerful predictor for cardiovascular events in univariate as well as multivariate analysis. Strain rate imaging is a very important tool for the evaluation of myocardial function [45]. Experimental studies have shown that strain rate is less dependent on LV load variations than strain [46]. Substantial potential limitations of speckle-tracking techniques are its strict dependence on the frame rate and on high-quality 2- dimensional images, which are necessary for obtaining an optimal definition of the endocardial border. However, because the strain rate signal is noisier and less reproducible, most clinical studies still use strain measurements [47]. According to previous studies, in the setting of global disease, in which the site-specificity of SR imaging in not required, there is no specific advantage in using SR instead of strain for assessing subclinical heart disease $[46,48,49]$.

The current study reports significantly higher BNP levels among all the patient groups compared to healthy controls, gradually increasing from the hypertensive group to the diabetic group, and ending up being the highest among the hypertensive diabetic group. BNP levels were correlated with LV strain \& strain rate values as well as values from conventional echocardiography \& TDI in all patients. These findings opposed the ones from Bakhoum et al [26], who stated that there was no significant difference in serum BNP level between patients \& control subjects.

But the current findings were consistent with the ones from Sadlecki et al [50], who stated that even though usually BNP levels are higher in pregnant women than in nonpregnant ones, the subjects recruited in his study showed that levels of BNP were significantly higher in pregnant women with gestational hypertension than normotensive pregnant ones. Current findings were also in line with findings from Hamed et al [40], who revealed that BNP levels were significantly higher among hypertensive patients compared to control subjects.

There was some disparity between our findings and ones from Wang et al [51], where the majority of diabetic patients included in the study had BNP levels within the normal range, despite their abnormal strain or diastolic dysfunction shown by echocardiography. Even with the noticed dispute among several studies, the negative predictive value of BNP cannot be denied, as it makes its assessment useful for excluding cardiac dysfunction in emergency settings with symptoms that may resemble those of cardiac origin [52].

Natriuretic peptides have been shown to be associated with left ventricular hypertrophy and cardiac functional abnormalities in individuals with chronic kidney disease without overt heart failure [53], as well as LV systolic and diastolic dysfunction in coronary artery disease [54]. Furthermore, prior studies have shown that BNP is increased in the setting of asymptomatic LV systolic dysfunction [55, 56].

\section{Study Limitations}

First, the study was based on echocardiographic techniques that are confined to a two-dimensional plane, and therefore ignores the complex motion in the third dimension, which causes the loss of acoustic speckles and less accurate results.

Second, recruited subjects had different onset, duration, and severity of either hypertension, diabetes, or both. The study was carried out on a small scale of patients, which may have influenced the results.

Finally, all measurements and examinations were performed while the patients were under their antihypertensive or anti-diabetic medications or both.

\section{Conclusions}

2D-STE is superior to conventional echocardiography; it can identify subclinical alterations of LV systolic \& diastolic functions in patients with arterial hypertension and glucose metabolism disorders.

Reduction of LV systolic function was detected in all 3 patient groups, manifested by the significant reduction of LV strain, even in the absence of reduced EF.

Hypertensive-diabetics exhibited significantly lower global strain than patients with hypertension only and patients with diabetes only, even though their EF showed no apparent difference.

LV diastolic deformation is prevalent among all patients, which was evidenced by the inverted E/A ratio and altered TDI parameters, in addition to the reduced peak strain rate at early diastole among the 3 patient groups.

Hypertensive diabetic patients had worse diastolic function than non-diabetic hypertensive patients and diabetic nonhypertensive patients.

Elevated BNP levels indicate the presence of ventricular dysfunction, even before chamber failure occurs.

The synergistic effects of diabetes and hypertension can induce more serious LV dysfunction. Their co-existence worsens the effect that each of them has on the LV when they exist alone.

\section{Abbreviations}

2D-STE: Two-dimensional Speckle Tracking
Echocardiography
A: Peak mitral flow A velocity
a': Peak mitral annular atrial velocity
ACC: American College of Cardiology
AHA: American Heart Association
AO: Aortic Root


BMI: Body mass index

BNP: B-type natriuretic peptide

CAD: Coronary Artery Disease

$\mathrm{CV}$ : Cardiovascular

CVD: Cardiovascular Disease

DM: Diabetes Mellitus

E: Peak early mitral flow velocity

e': Peak early mitral annular velocity

ECG: Electrocardiogram

ELISA: Enzyme-linked immunosorbent assay

ESC: European Society of Cardiology

ESH: European Society of Hypertension

GLS: Global Longitudinal Strain

HF: Heart Failure

HHD: Hypertensive Heart Disease

HTN: Hypertension

IVSD: End-diastolic thickness of the ventricular septum

LA: Left Atrial

LV: Left Ventricular

LVEDd: The end-diastolic LV diameter

LVEDP: Left Ventricular End Diastolic Pressure

LVEDs: End-systolic LV diameter

LVEF: Left Ventricular Ejection Fraction

NP: Natriuretic Peptide

NPR: Natriuretic Peptide Receptors

NT-Pro BNP: N-Terminal fragment of B-type natriuretic peptide

PVC: Premature Ventricular Contraction

PWd: End-diastolic thickness of the LV posterior wall

SR: Strain rate

STE: Speckle-tracking echocardiography

TDI: Tissue Doppler Imaging

\section{References}

[1] Ilias Karabinos, Charalampos Grassos, Panajiota Kostaki, Athanasios Kranidis (2013) Echocardiography in the evaluation of hypertensive patient: An invaluable tool or simply following the routine? Hellenic J. Cariol. 54: 47-57.

[2] Shi Y, Hu FB (2014) The global implications of diabetes and cancer. Lancet 383: 1947-1948.

[3] Dodiyi-Manuel ST, Akpa MR, Odia OJ (2013) Left ventricular dysfunction in normotensive type II diabetic patients in Port Harcourt, Nigeria. Vasc Health Risk Manag. 9: 529-533.

[4] Landsberg L, Molitch M (2004) Diabetes and hypertension: pathogenesis, prevention and treatment. Clin Exp Hypertens. 26: 621-8.

[5] Bernard M. Y. Cheung, Nelson M. S. Wat, Annette W. K. Tso, Sidney Tam, G. Neil Thomas, Gabriel M. Leung, Hung Fat Tse, Jean Woo, Edward D. Janus, Chu Pak Lau, et al (2008). Association between raised blood pressure and dysglycemia in Hong Kong Chinese. Diabetes Care 31: 1889-91.

[6] Bernard M. Y. Cheung and Chao Li (2012) Diabetes and Hypertension: Is There a Common Metabolic Pathway? Curr Atheroscler Rep 14: 160-166.
[7] Kannel WB, Hjortland M, Castelli WP (1974) Role of diabetes in congestive heart failure: the Framingham study. Am J Cardiol. 34: 29-34.

[8] Grossman E, Messerli FH (1996) Diabetic and hypertensive heart disease. Ann Intern Med. 125: 304-310.

[9] Gottdiener JS, Arnold AM, Aurigemma GP, Polak JF, Tracy RP, Kitzman DW, Gardin JM, Rutledge JE, Boineau RC (2000) Predictors of congestive heart failure in the elderly: the Cardiovascular Health Study. J Am Coll Cardiol. 35: 16281637.

[10] Dominick M. Carella (2015) Brain Natriuretic Peptide: It's Not About the Brain or Just Another Smart Polypeptide-It's About the Heart. Neonatal network. 34: 6.

[11] Hammerer-Lercher A, Neubauer E, Muller S, Pachinger O, Puschendorf B, Mair J (2001) Head-to-head comparison of N terminal pro-brain natriuretic peptide, brain natriuretic peptide and N-terminal pro-atrial natriuretic peptide in diagnosing left ventricular dysfunction. Clin Chim Acta. 310: 193-197.

[12] Ritchie RH, Rosenkranz AC, Kaye DM (2009) B-type natriuretic peptide: endogenous regulator of myocardial structure, biomarker and therapeutic target. Curr Mol Med. 9 (7): 814-25.

[13] A. Battistoni, S. Rubattu, M. Volpe (2012) Circulating biomarkers with preventive, diagnostic and prognostic implications in cardiovascular diseases. Int. J. Cardiol. 157: 160-168.

[14] Kazuhiro Yamamoto, John C. BurnettJr, Michihisa Jougasaki, Rick A. Nishimura, Kent R. Bailey, Yoshihiko Saito, Kazuwa Nakao, and Margaret M. Redfield (1996) Superiority of Brain Natriuretic Peptide as a Hormonal Marker of Ventricular Systolic and Diastolic Dysfunction and Ventricular Hypertrophy. Hypertension 28: 988-994.

[15] de Lemos JA, Morrow DA, Bentley JH (2001) The prognostic value of B-type natriuretic peptide in patients with acute coronary syndromes. N Engl J Med. 345: 1014-1021.

[16] P. Gæde, P. Hildebrandt, G. Hess, H.-H. Parving, O. Pedersen (2005) Plasma N-terminal pro-brain natriuretic peptide as a major risk marker for cardiovascular disease in patients with type 2 diabetes and microalbuminuria. Diabetologia 48: 156-163.

[17] Kim DH, Kim HK, Kim MK, Chang SA, Kim YJ, Kim MA, Sohn DW, Oh BH, Park YB (2009) Velocity vector imaging in the measurement of left ventricular twist mechanics: head-tohead one way comparison between speckle tracking echocardiography and velocity vector imaging. J Am Soc Echocardiogr. 22: 1344-52.

[18] Liu YW, Tsai WC, Su CT, Lin CC, Chen JH (2009) Evidence of left ventricular systolic dysfunction detected by automated function imaging in patients with heart failure and preserved left ventricular ejection fraction. J Card Fail. 15: 782-789.

[19] Sanderson JE (2007) Heart failure with a normal ejection fraction. Heart 93: 155-158.

[20] Sharon Ann Hunt, William T. Abraham, Marshall H. Chin, Arthur M. Feldman, Gary S. Francis, Theodore G. Ganiats, Mariell Jessup, Marvin A. Konstam, Donna M. Mancini, Keith Michl, et al (2009) 2009 Focused Update Incorporated Into the ACC/AHA 2005 Guidelines for the Diagnosis and Management of Heart Failure in Adults. J Am Coll Cardio. 14: e1-e90. 
[21] Mizuguchi Y, Oishi Y, Miyoshi H, Iuchi A, Nagase N, Oki T (2010) Concentric left ventricular hypertrophy brings deterioration of systolic longitudinal, circumferential, and radial myocardial deformation in hypertensive patients with preserved left ventricular pump function. J Cardiol. 55: 23-33.

[22] Nakai H, Takeuchi M, Nishikage T, Lang RM, Otsuji Y (2009) Subclinical left ventricular dysfunction in asymptomatic diabetic patients assessed by two-dimensional speckle tracking echocardiography: correlation with diabetic duration. Eur J Echocardiogr. 10: 926-932.

[23] Lang RM, Bierig M, Devereux RB, Flachskampf FA, Foster E, Pellikka PA, Picard MH, Roman MJ, Seward J, Shanewise JS, Solomon SD, Spencer KT, Sutton MS, Stewart WJ (2005) Recommendations for chamber quantification: a report from the American Society of Echocardiography's Guidelines and Chamber Quantification Writing Group, developed in conjunction with the European Association of Echocardiography, a branch of the European Society of Cardiology. J Am Soc Echocardiogr. 18: 1440-63.

[24] Richard FM, Richard H and Robert JM (1996) A study guide to epidemiology and biostatistics. 4th Ed. Gaithersburg, Md.: Aspen Publishers, 1996.

[25] Wang Q, Gao Y, Tan K, Xia H, and Li B (2015) Assessment of left ventricular function by three-dimension speckle-tracking echocardiography in well-treated type 2 diabetes patients with or without hypertension. J Clin Ultrasound 43: 502-511.

[26] Bakhoum S W G, Habeeb H A, Elebrashy I N, and Rizk M N (2016) Assessment of left ventricular function in young type 1 diabetes mellitus patients by two-dimensional speckle tracking echocardiography: Relation to duration and control of diabetes. The Egyptian Heart Journal 68 (4): 217-225.

[27] Stevanovic A and Dekleva M (2018) The importance of subclinical left ventricular dysfunction and blood pressure pattern in asymptomatic type 2 diabetic patients: the diagnostic and prognostic significance of tissue Doppler parameters, left ventricular global longitudinal strain, and nighttime blood pressure during sleep. Journal of Diabetes and its Complications 32: 41-47.

[28] Soufi-Taleb N, Tani A M (2016) Impact of diabetes and hypertension on the longitudinal systolic function of the left ventricle. Archives of Cardiovascular Diseases Supplements 8 (1): 81-82 DOI : 10.1016/S1878-6480(16)30232-4.

[29] Zhang X, Wei X, Liang Y, Liu M, Li C, and Tang H (2013) Differential changes of left ventricular myocardial deformation in diabetic patients with controlled and uncontrolled blood glucose: a three-dimensional speckletracking echocardiography-based study. J Am Soc $\begin{array}{lllll}\text { Echocardiogr. } & 26 & \text { (5): } & 499-506 . & \text { DOI }\end{array}$ 10.1016/j.echo.2013.02.016.

[30] Ikonomidis I, Tzortzis S, Triantafyllidi H, Parissis J, Papadopoulos C, Venetsanou K, Trivilou P, Paraskevaidis I, and Lekakis J (2015) Association of impaired left ventricular twisting-untwisting with vascular dysfunction, neurohumoral activation and impaired exercise capacity in hypertensive heart disease. Eur J Heart Fail. 17 (12): 1240-51. doi: 10.1002/ejhf.403. Epub 2015 Oct 7.

[31] Kolesnyk M. Yu (2014) Speckle tracking echocardiography in hypertensive males with glucose metabolism disorders. Zaporozhye Medical Journal 6 (87): 4-10.

[32] Ernande L, Bergerot C, Girerd N, Thibault H, Davidsen ES,
Gautier Pignon-Blanc P, Amaz C, Croisille P, De Buyzere ML, Rietzschel ER, Gillebert TC, Moulin P, Altman M, Derumeaux G (2014) Longitudinal myocardial strain alteration is associated with left ventricular remodeling in asymptomatic patients with type 2 diabetes mellitus. J Am Soc $\begin{array}{llll}\text { Echocardiogr. } & 27 & \text { (5): } & 479-88\end{array}$ 10.1016/j.echo.2014.01.001

[33] Yau-Huei Lai, Chi-In Lo, Yih-Jer Wu, Chung-Lieh Hung, and Hung-I Yeh (2013). Cardiac Remodeling, Adaptations and Associated Myocardial Mechanics in Hypertensive Heart Diseases. Acta Cardiol Sin 29 (1): 64-70.

[34] Imbalzano E, Zito C, Carerj S, Oreto G, Mandraffino G, Cusma-Piccione M, Di Bella G, Saitta C, and Saitta A (2011) Left Ventricular Function in Hypertension: New Insight by Speckle Tracking Echocardiography. Echocardiography 28 (6): 649-657 DOI: 10.1111/j.1540-8175.2011.01410.x.

[35] Li T, Chen S, Guo X, Yang J, Sun Y (2017) Impact of hypertension with or without diabetes on left ventricular remodeling in rural Chinese population: a cross-sectional study. BMC Cardiovasc Disord. 17 (1): 206. doi: 10.1186/s12872-017-0642-y.

[36] Ernande L, Bergerot C, Rietzschel ER, De Buyzere ML, Thibault H, Pignonblanc PG, Croisille P, Ovize M, Groisne L, Moulin P, Gillebert TC, and Derumeaux G (2011) Diastolic dysfunction in patients with type 2 diabetes mellitus: is it really the first marker of diabetic cardiomyopathy? J Am Soc Echocardiogr. 24 (11): 1268-1275. doi: 10.1016/j.echo.2011.07.017.

[37] Lumens J, Delhaas T, Arts T, Cowan BR, Young AA (2006) Impaired subendocardial contractile myofiber function in asymptomatic aged humans, as detected using MRI. Am J Physiol Heart Circ Physiol 291 (4): H1573-9.

[38] Nikitin NP, Witte KK, Thackray SD, de Sileft ventriculara R, Clark AL, Cleland JG (2003) Longitudinal ventricular function: normal values of atrioventricular annular and myocardial velocities measured with quantitative twodimensional color Doppler tissue imaging. J Am Soc Echocardiogr 16 (9): 906-21.

[39] López B, Querejeta R, González A, Sánchez E, Larman M, Díez J (2004) Effects of loop diuretics on myocardial fibrosis and collagen type I turnover in chronic heart failure. J Am Coll Cardiol. 43: 2028-35.

[40] Hamed W A I, Kamal A M, Noamany M F, Soliman M A, and AbdelRa'ouf M M (2014) Evaluation of left ventricular performance in hypertensive patients by speckle tracking echocardiography: Correlation with brain natriuretic peptide. The Egyptian Heart Journal 66: 299-308.

[41] Bonito P, Moio N, Cavuto L, Covino G, Murena E, Scilla C, Turco S, Capaldo B, Sibilio G. (2005) Early detection of diabetic cardiomyopathy: usefulness of tissue Doppler imaging. Diabet Med 22: 1720.

[42] Von Bibra H, Thrainsdottir IS, Hansen A, Dounis V, Malmberg K, Rydén L. (2005) Tissue Doppler imaging for the detection and quantitation of myocardial dysfunction in patients with type 2 diabetes mellitus. Diab Vasc Dis Res 2: 24.

[43] J. D'hooge, A. Heimdal, F. Jamal, T. Kukulski, B. Bijnens, F. Rademakers, L. Hatle, P. Suetens, G. R. Sutherland (2000) Regional strain and strain rate measurements by cardiac ultrasound: principles, implementation and limitations. Eur $\mathbf{J}$ Echocardiogr 1: 154. 
[44] Helle-Valle, Crosby J, Edvardsen T, Lyseggen E, Amundsen BH, Smith HJ, Rosen BD, Lima JA, Torp H, Ihlen H, Smiseth OA. (2005) New noninvasive method for assessment of left ventricular rotation: speckle tracking echocardiography. Circulation 112: 3149 .

[45] Dandel M, Lehmkuhl H, Knosalla C, Suramelashvili N, Hetzer R (2009) Strain and strain rate imaging by echocardiography - basic concepts and clinical applicability. Curr Cardiol Rev. 5: 133-48.

[46] Marwick TH (2006) Measurement of strain and strain rate by echocardiography ready for prime time? J Am Coll Cardiol. 47: 1313-27.

[47] Mondillo S, Galderisi M, Mele D, Cameli M, Lomoriello VS, Zacà Z (2011) Speckle-tracking echocardiography: a new technique for assessing myocardial function. J Ultrasound Med. 30: 71-83.

[48] Fang ZY, Yuda S, Anderson V, Short L, Case C, Marwick TH (2003) Echocardiographic detection of early diabetic myocardial disease. J Am Coll Cardiol. 41: 611-7.

[49] Geyer H, Caracciolo G, Abe H, Wilansky S, Carerj S, Gentile F (2010) Assessment of myocardial mechanics using speckle tracking echocardiography: fundamentals and clinical applications. J Am Soc Echocardiogr. 23: 351-69.

[50] Sadlecki P, Grabiec M, and Walentowicz-Sadlecka M (2016) Prenatal clinical assessment of nt-probnp as a diagnostic tool for preeclampsia, gestational hypertension and gestational diabetes mellitus. PLoS One 11 (9): e0162957. doi: 10.1371/journal.pone.0162957.
[51] Wang TJ, Larson MG, Keyes MJ, Levy D, Benjamin EJ, and Vasan RS (2007) Association of plasma natriuretic peptide levels with metabolic risk factors in ambulatory individuals. Circulation 115 (11): 1345-53.

[52] Abd-El Raof M. M (2014) Evaluation of left ventricular performance in hypertensive patients by speckle tracking echocardiography: correlations with brain natriuretic peptide level. M. Sc. Dissertation, Faculty of Medicine, Menoufia University.

[53] Mishra RK, Li Y, Ricardo AC, Yang W, Keane M, Cuevas M, Christenson R, DeFilippi C, Chen J, He J, Kallem RR, Raj DS, Schelling JR, Wright J, Go AS, Shlipak MG (2013) Association of N-Terminal pro-B-type natriuretic peptide with left ventricular structure and function in chronic kidney disease (from the Chronic Renal Insufficiency Cohort [CRIC]). Am J Cardiol 111: 432-438.

[54] Sonoda H, Ohte N, Goto T, Wakami K, Fukuta H, Kikuchi S, Tani T, Kimura G (2012) Plasma N-terminal pro-brain natriuretic peptide levels identifying left ventricular diastolic dysfunction in patients with preserved ejection fraction. Circ $\mathrm{J}$ 76: 2599-2605.

[55] McDonagh T, Robb S, Murdoch D, Morton J, Ford I, Morrison C, Tunstall-Pedoe H, McMurray JJV, Dargie HJ (1998) Biochemical detection of left-ventricular systolic dysfunction. Lancet 351: 9-13.

[56] Troughton RW, Richards AM (2009) B-type natriuretic peptides and echocardiographic measures of cardiac structure and function. JACC: Cardiovascular Imaging 2: 216-225. 